\title{
A Comparative Study on Skin and Plasma Advanced Glycation End Products and Their Associations with Arterial Stiffness
}

\author{
Chang-Yuan Liu Qi-Fang Huang Yi-Bang Cheng Qian-Hui Guo \\ Qi Chen Yan Li Ji-Guang Wang
}

Centre for Epidemiological Studies and Clinical Trials, Shanghai Key Laboratory of Hypertension, The Shanghai Institute of Hypertension, Department of Hypertension, Ruijin Hospital, Shanghai Jiaotong University School of Medicine, Shanghai, China

\section{Keywords}

Advanced glycation end products · Skin autofluorescence · Plasma concentration · Diabetes . Prediabetes · Arterial stiffness · Carotid-femoral pulse wave velocity

\begin{abstract}
Background: We compared skin and plasma measurements of advanced glycation end products (AGEs), with particular focus on their levels in the presence of hypertension or diabetes and prediabetes and their associations with arterial stiffness in outpatients with suspected or diagnosed hypertension. Methods: Skin AGE accumulation was measured as autofluorescence on the left forearm using the skin autofluorescence Reader and expressed in arbitrary units in the range from 0 to 25 . Plasma AGE concentration was measured by the enzymelinked immunosorbent assay method and logarithmically transformed for statistical analysis. Arterial stiffness was assessed by carotid-femoral pulse wave velocity (cfPWV) using the SphygmoCor system (Sydney, Australia). Results: The 218 participants (96 [44.0\%] men, mean age 51.9 years) had a mean skin autofluorescence of 1.89 arbitrary units, plasma AGE concentration of $4.47 \mu \mathrm{g} / \mathrm{ml}$, and cfPWV of $8.0 \mathrm{~m} / \mathrm{s}$. Skin autofluorescence was significantly correlated with plasma AGEs in diabetic or prediabetic patients $(n=31, r=0.37, p=0.04)$ but not in subjects with normoglycemia $(n=187, r=-0.05, p=0.48)$. Nonetheless, both measurements were significantly $(p \leq 0.001)$ higher in men $(2.00$ arbitrary units and $6.73 \mu \mathrm{g} / \mathrm{ml}$, respectively) than women (1.81 arbitrary units and $3.60 \mu \mathrm{g} / \mathrm{ml}$, respectively) and in diabetic or prediabetic (2.03 arbitrary units and $6.61 \mu \mathrm{g} / \mathrm{ml}$, respectively) than normoglycemia subjects (1.87 arbitrary units and $4.17 \mu \mathrm{g} / \mathrm{ml}$, respectively), but similar in hypertensive $(n=105)$ and normotensive subjects $(n=113, p \geq 0.35)$. In adjusted multiple regression analyses, plasma AGE concentration, but not skin autofluorescence $(p \geq 0.37)$, was significantly associated with cfPWV in all subjects ( $\beta 0.44 \mathrm{~m} / \mathrm{s}$ for each 10 -fold increase; $p=0.04$ ) and in subgroups of men
\end{abstract}


Liu et al.: A Comparative Study on Skin and Plasma Advanced Glycation End Products and Their Associations with Arterial Stiffness

and diabetes and prediabetes ( $\beta 0.12-0.55 \mathrm{~m} / \mathrm{s}$ for each 10 -fold increase; $p \leq 0.02$ ). Conclusions: Although skin and plasma AGEs were similarly associated with gender and diabetes or prediabetes, they might measure something different and have different clinical relevance, such as for arterial stiffness.

(C) 2016 S. Karger AG, Basel

\section{Introduction}

Advanced glycation end products (AGEs) are a heterogeneous group of bioactive compounds endogenously generated by a nonenzymatic reaction between reducing sugars and amine residues on proteins, lipids, or nucleic acids $[1,2]$ or exogenously inhaled from cigarette smoking [3] or ingested from diets [4]. Several previous studies showed that AGE accumulation may be clinically important in the pathogenesis, prediction, and prevention of cardiovascular-metabolic disease [5-7].

AGEs are usually assessed biochemically by measuring plasma concentration of one or more components of AGEs, such as pentosidine, carboxyethyl lysine, $\mathrm{N}$-carboxymethyl lysine (CML), etc. [1]. These biochemical measurements were found to be correlated with arterial properties [8-10] and predictive of cardiovascular events [5-7, 11]. Indeed, we previously found in our general population cohort that plasma AGE concentration was higher with advancing age and with higher serum total-to-high-density lipoprotein (HDL) cholesterol ratio [9], and was associated with arterial wave reflections [9], pressure amplification [12], and arterial stiffness [9]. However, circulating AGEs are not only dependent on the endogenous production but also on the ingestion [4] and excretion [13]. Therefore, it may not accurately reflect the accumulation in tissues. Certain dermal AGEs possess autofluorescent properties, such as pentosidine [14]. The measurement of skin autofluorescence may behave as a marker of tissue accumulation of AGEs. A device was developed with a proprietary algorithm for the assessment of forearm skin autofluorescence as a measure of tissue AGE accumulation [14]. The device allows rapid, low-cost, noninvasive estimation of AGE accumulation and has been validated in a skin biopsy study of healthy subjects [14].

Recent studies with this device measurement alone suggested that skin autofluorescence was associated with intermediate [15-17] and clinical cardiovascular outcomes [18, 19]. However, the device-based skin autofluorescence and biochemical plasma measurements of AGEs have been rarely investigated simultaneously for their clinical relevance with regard to associations with known cardiovascular risk factors and arterial stiffness [20]. In the present study, we compared skin autofluorescence and plasma AGE concentration with particular focus on their levels in the presence of hypertension or diabetes and prediabetes, and their associations with arterial stiffness as assessed by carotid-femoral pulse wave velocity (cfPWV).

\section{Methods}

Study Population

Our study subjects were consecutive patients who were referred for ambulatory blood pressure monitoring to Ruijin Hospital, Shanghai, China $[21,22]$. The study protocol was approved by the Ethics Committee of Ruijin Hospital, Shanghai Jiaotong University School of Medicine. All participants gave written informed consent. Between July 2013 and June 2015, 243 patients were enrolled in the study of skin autofluorescence and plasma AGE concentration. We excluded 25 participants from the present analysis because their skin autofluorescence $(n=13)$ and/or plasma AGE concentration $(n=17)$ could not be successfully measured. Thus, the total number of participants included in the present analysis was 218 . 


\section{Skin Autofluorescence and Plasma AGE Concentration}

Skin autofluorescence was assessed using the skin autofluorescence Reader (DiagnOptics Technologies BV, Groningen, The Netherlands). This noninvasive method is completely automated. The autofluorescence Reader illuminates a skin surface of $1 \mathrm{~cm}^{2}$ with an excitation light source of 300-420 nm. The emission light and the reflected excitation light from the skin are measured using a spectrometer (model PC-1000 fiber optic spectrometer; Ocean Optics, Dunedin, FL, USA). Autofluorescence is calculated as the average light intensity per nanometer in the range between 420 and $600 \mathrm{~nm}$, divided by the average light intensity per nanometer in the range between 300 and $420 \mathrm{~nm}$, multiplied by 100 and expressed in arbitrary units in the range from 0 to 25 .

In our study, a series of 3 consecutive measurements were performed on both forearms $(n=76)$ or on the left forearm only $(n=142)$ at about $5 \mathrm{~cm}$ distal to the elbow fold. The whole measurement process took less than a minute. Mean skin autofluorescence was calculated from the 3 consecutive measurements on the left arm and used for the analyses. In our reproducibility study $(n=39)$, the intrasubject, intersession coefficient of variation was $11.2 \%$, slightly higher than previously reported (5\%) [14].

Plasma AGE concentration was measured by the enzyme-linked immunosorbent assay method (AGEs ELISA Kit, Cusabio Biotech, Wilmington, DE, USA) as described previously [9, 12]. The intra- and interassay coefficients of variation were 9.9 and $10.9 \%$, respectively.

\section{Carotid-Femoral PWV}

cfPWV was measured by applanation tonometry (Millar tonometer: SPC-301; Millar Instruments, Houston, TX, USA). Subjects were asked to refrain from smoking, drinking alcohol or caffeine containing beverages and rigorous exercise for at least $2 \mathrm{~h}$ before the examination. The arterial waveforms were sequentially recorded at the carotid and femoral arterial sites using the SphygmoCor device (AtCor Medical, West Tyde, NSW, Australia) after the subjects had rested for about $10 \mathrm{~min}$ in the supine position. With the simultaneously recorded electrocardiogram (lead II), the time delay between the foot of the two pressure waveforms was taken as the transit time between carotid and femoral arterial sites. The distance traveled by the pressure wave was the difference between the distances from sternal notch to the femoral location and from sternal notch to the carotid location. cfPWV was calculated as the distance traveled divided by the transit time. The intraobserver coefficient of variability was $2.5 \%$ for the cfPWV.

\section{Questionnaire, Anthropometry, and Other Clinical and Laboratory Measurements}

A trained technician administered a standardized questionnaire to collect information on each participant's medical history, smoking and drinking habits, and use of medications. Body height was measured to the nearest $0.5 \mathrm{~cm}$. Body weight was measured while they wore light indoor clothing without shoes for measurement. Body mass index was calculated as the body weight in kilograms divided by the body height in meters squared.

Clinic blood pressure was measured by an experienced physician with the Omron HEM-7051 monitor (Omron HealthCare, Kyoto, Japan). After the participants had rested for at least 5 min in the sitting position, 3 consecutive blood pressure readings were obtained according to the recommendations of the European Society of Hypertension [23]. These 3 blood pressure readings were averaged for analysis. Hypertension was defined as a blood pressure of at least $140 \mathrm{~mm} \mathrm{Hg}$ systolic or $90 \mathrm{~mm} \mathrm{Hg}$ diastolic.

Venous blood samples were drawn after overnight fasting for biochemical measurements. Plasma glucose concentration and serum total and HDL cholesterol and serum triglycerides were measured using a clinical chemistry automatic analyzer (Hitachi 7600020, Tokyo, Japan). Diabetes mellitus was defined as a plasma glucose concentration of at least $7.0 \mathrm{mmol} / \mathrm{L}$ fasting or $11.1 \mathrm{mmol} / \mathrm{L}$ at any time or as the use of antidiabetic agents. Prediabetes was defined as a plasma fasting glucose in the range of 6.1-6.9 mmol/L.

\section{Statistical Analysis}

For database management and statistical analysis, we used the SAS software (version 9.2; SAS Institute, Cary, NC, USA). Departure from normality was tested by the Shapiro-Wilk statistic. Plasma AGE concentration was logarithmically transformed for statistical analysis. For comparison of means and proportions, we performed the Student $t$ test and the $\chi^{2}$ test, respectively. We performed stepwise multiple regression analyses to search for possible correlates of skin autofluorescence and plasma AGE concentration with the $p$ value for covariables to enter and stay in the model set at 0.10 . We assessed the interrelationship between skin autofluorescence and plasma AGE concentration, while accounting for the confounding factors. We also performed multiple regression analyses to study associations of skin autofluorescence and plasma AGE concentration with cfPWV. 


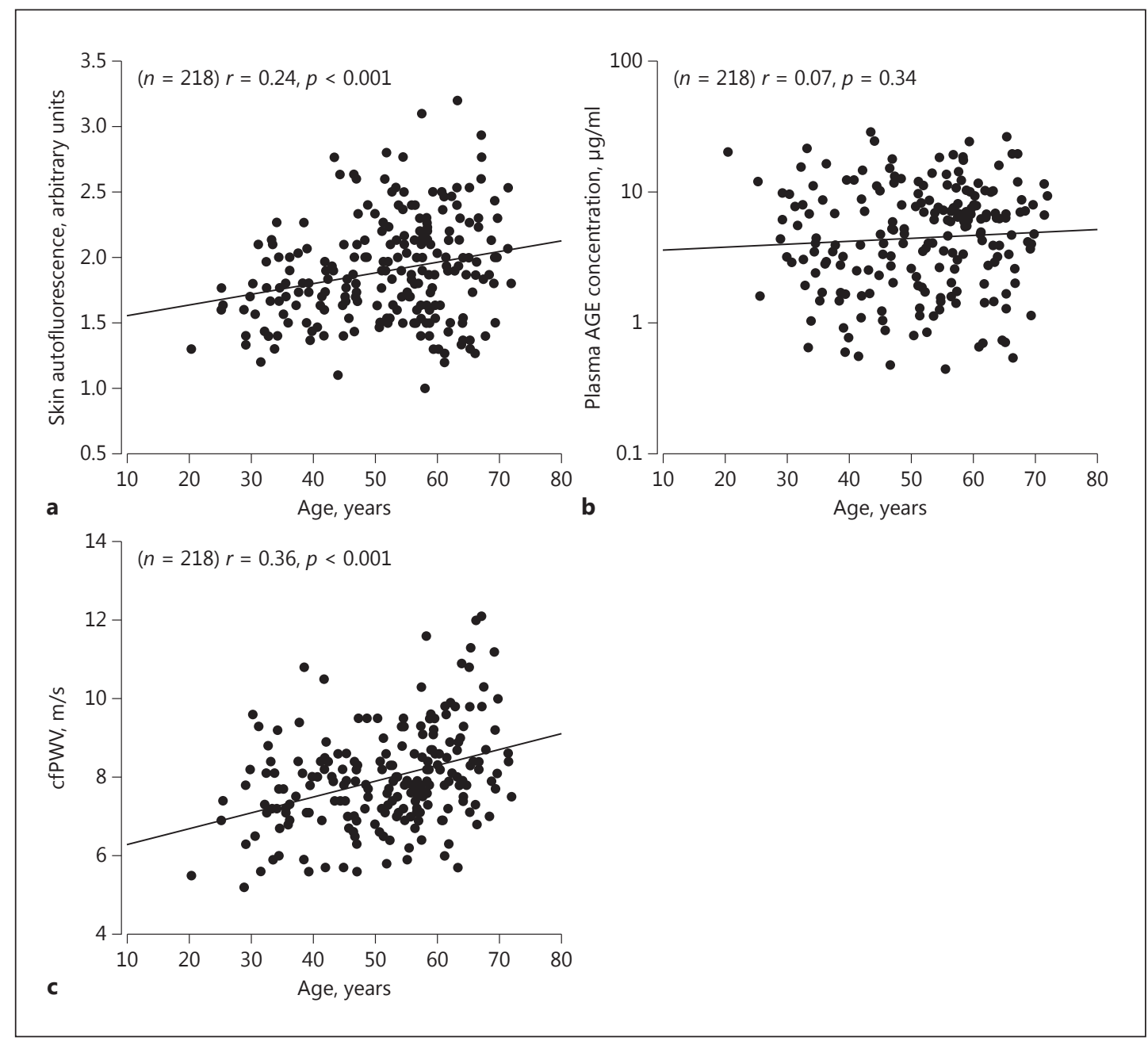

Fig. 1. Skin autofluorescence (a), plasma advanced glycation end product (AGE) concentration (b), and carotid-femoral pulse wave velocity (cfPWV) (c) in relation to age. A regression line was drawn. Correlation coefficients and their $p$ values are given.

\section{Results}

\section{Characteristics of the Study Population}

The 218 participants included 96 (44.0\%) men and 105 (48.2\%) hypertensive patients who did not take any antihypertensive medication. Men, compared with women, were younger $(-4.5$ years, $p=0.01)$ and had a significantly $(p<0.001)$ greater body mass index $(+1.5)$, higher proportions of current smoking (31.9 vs. $0.8 \%$ ) and alcohol intake (34.0 vs. $3.4 \%)$, and higher levels of diastolic blood pressure $(+2.7 \mathrm{~mm} \mathrm{Hg})$, serum total-to-HDL ratio $(+0.40)$ and triglycerides $(+0.36 \mathrm{mmol} / \mathrm{L})$, and serum creatinine $(+16.7 \mu \mathrm{mol} / \mathrm{L})$ and uric acid $(+80.7 \mu \mathrm{mol} / \mathrm{L})$. They had similar $(p \geq 0.15)$ systolic blood pressure $(136.9 \mathrm{~mm} \mathrm{Hg})$, pulse rate $(72.5$ beat/min), prevalence of hypertension $(52.5 \%)$ and diabetes or prediabetes (15.5\%), fasting plasma glucose $(5.33 \mathrm{mmol} / \mathrm{L})$, and serum total cholesterol $(5.55 \mathrm{mmol} / \mathrm{L})$.

Men, compared with women, had significantly $(p \leq 0.001)$ higher levels of skin autofluorescence ( 2.00 vs. 1.81 arbitrary units) and plasma AGE concentration ( 6.73 vs. $3.60 \mu \mathrm{g} / \mathrm{mL})$. 
Fig. 2. Scatter plot for the correlation between skin autofluorescence and plasma advanced glycation end product (AGE) concentration in subjects with diabetes or prediabetes.
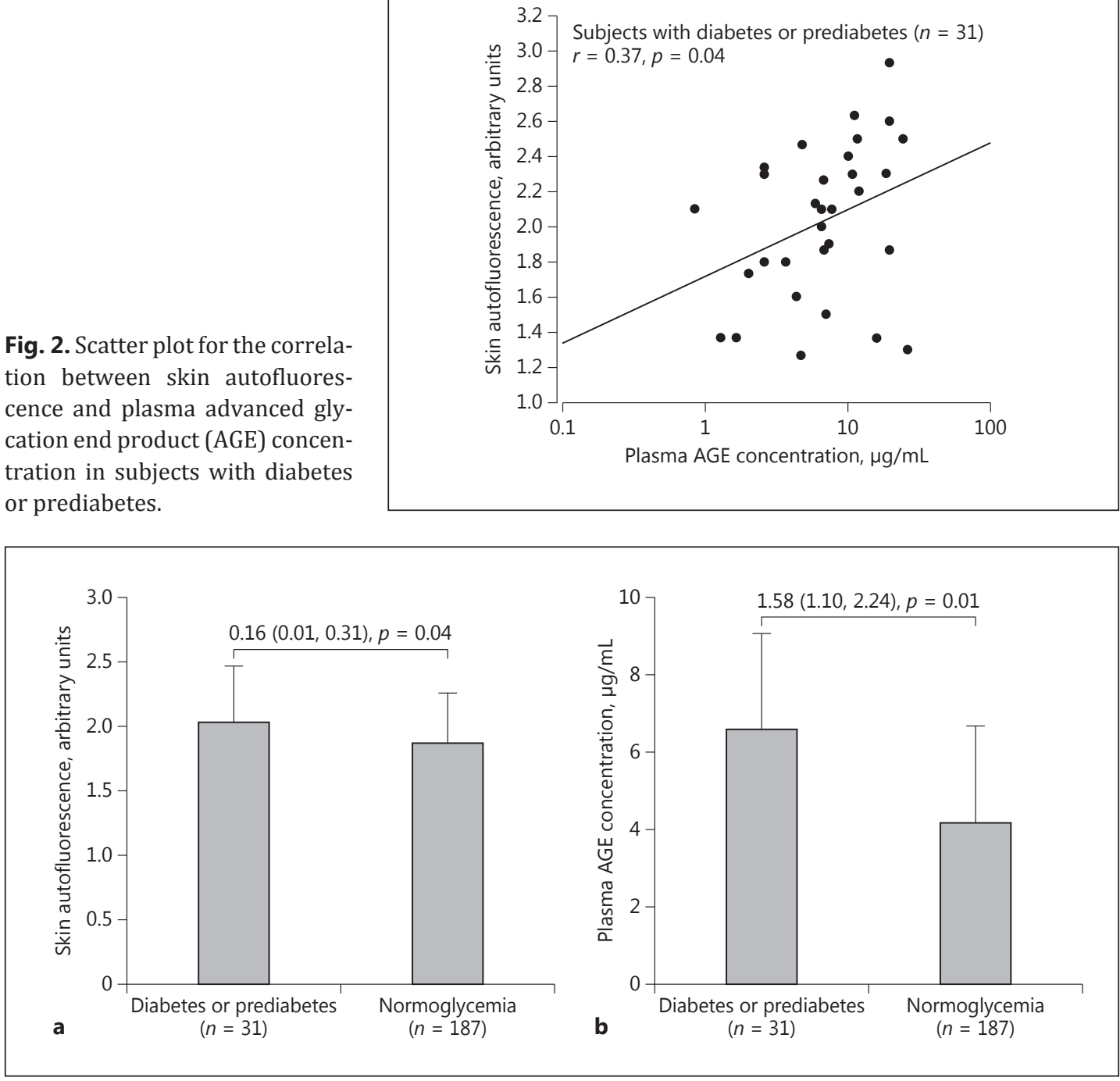

Fig. 3. Skin autofluorescence (a) and plasma advanced glycation end product (AGE) concentration (b) according to the presence or absence of diabetes or prediabetes. Differences ( $95 \%$ confidence interval) between patients with diabetes or prediabetes and those without and their $p$ values are given.

They had similar cfPWV (7.97 vs. $7.98 \mathrm{~m} / \mathrm{s}, p=0.94)$. Skin autofluorescence $(r=0.24, p<$ $0.001)$ and cfPWV ( $r=0.36, p<0.001)$, but not plasma AGE concentration $(r=0.07, p=0.34)$, were significantly higher with advancing age (Fig. 1).

\section{Interrelationship between Skin Autofluorescence and Plasma AGE Concentration}

Skin autofluorescence was significantly correlated with plasma AGE concentration in diabetic or prediabetic patients $(n=31, r=0.37, p=0.04$; Fig. 2$)$ but not nondiabetic subjects $(n=187, r=-0.05, p=0.48)$. Nonetheless, both AGE measurements were significantly ( $p \leq$ $0.04)$ higher in the presence of diabetes or prediabetes $(2.03$ arbitrary units and $6.61 \mu \mathrm{g} / \mathrm{ml}$, respectively) than normoglycemia (1.87 arbitrary units and $4.17 \mu \mathrm{g} / \mathrm{ml}$, respectively; Fig. 3).

In further analyses, we first categorized the study subjects into four groups according to the median levels of the two AGE measurements (Table 1). These 4 groups differed signifi- 
Liu et al.: A Comparative Study on Skin and Plasma Advanced Glycation End Products and Their Associations with Arterial Stiffness

Table 1. Characteristics of the study population according to the median levels of skin autofluorescence and plasma concentration of AGEs

\begin{tabular}{|c|c|c|c|c|c|}
\hline Characteristic & $\begin{array}{l}\text { Both skin AF } \\
\text { and plasma AGEs } \\
\text { below median } \\
(n=61)\end{array}$ & $\begin{array}{l}\text { Skin AF below } \\
\text { median and plasma } \\
\text { AGEs above median } \\
(n=47)\end{array}$ & $\begin{array}{l}\text { Skin AF above } \\
\text { median and plasma } \\
\text { AGEs below median } \\
(n=47)\end{array}$ & $\begin{array}{l}\text { Both skin AF and } \\
\text { plasma AGEs above } \\
\text { median } \\
(n=63)\end{array}$ & $\begin{array}{l}p \\
\text { (ANOVA) }\end{array}$ \\
\hline Age, years & $47.4 \pm 11.6$ & $50.3 \pm 13.2$ & $54.7 \pm 9.8^{*}$ & $55.2 \pm 9.8^{*}$ & $<0.0001$ \\
\hline Men & $18(29.5)$ & $20(42.6)$ & $17(36.2)$ & $41(65.1)^{*}$ & $<0.0001$ \\
\hline Body mass index & $24.9 \pm 3.5$ & $24.4 \pm 3.1$ & $24.1 \pm 2.6$ & $24.8 \pm 3.3$ & 0.53 \\
\hline Current smoking & $7(11.5)$ & $2(4.3)$ & $8(17.0)^{\dagger}$ & $14(22.2)^{*}$ & 0.04 \\
\hline Alcohol intake & $5(8.2)$ & $6(12.8)$ & $10(21.3)$ & $15(23.8)^{*}$ & 0.01 \\
\hline Systolic blood pressure, $\mathrm{mm} \mathrm{Hg}$ & $136.8 \pm 15.5$ & $137.4 \pm 14.3$ & $136.5 \pm 17.1$ & $137.3 \pm 13.2$ & 0.99 \\
\hline Diastolic blood pressure, $\mathrm{mm} \mathrm{Hg}$ & $84.9 \pm 11.4$ & $84.7 \pm 11.7$ & $81.4 \pm 9.9$ & $84.2 \pm 9.7$ & 0.34 \\
\hline Pulse rate, beats $/ \mathrm{min}$ & $72.9 \pm 10.0$ & $73.8 \pm 9.9$ & $69.3 \pm 10.6^{\dagger}$ & $73.0 \pm 10.8$ & 0.15 \\
\hline Prevalence of hypertension & $31(50.8)$ & $25(53.2)$ & $20(42.6)$ & $29(46.0)$ & 0.72 \\
\hline Fasting plasma glucose, $\mathrm{mmol} / \mathrm{L}$ & $5.3 \pm 1.1$ & $5.2 \pm 0.5$ & $5.3 \pm 0.7$ & $5.5 \pm 0.9$ & 0.51 \\
\hline Prevalence of diabetes or & & & & & \\
\hline prediabetes & 7( & 3 & 4 & $17(27.0)^{*}$ & 0.01 \\
\hline Serum total cholesterol, $\mathrm{mmol} / \mathrm{L}$ & $5.47 \pm 1.05$ & $5.37 \pm 1.16$ & $5.56 \pm 1.11$ & $5.79 \pm 1.12$ & 0.22 \\
\hline Serum HDL cholesterol, mmol/L & $1.51 \pm 0.43$ & $1.44 \pm 0.34$ & $1.57 \pm 0.38$ & $1.51 \pm 0.54$ & 0.55 \\
\hline Serum total/HDL cholesterol ratio & $3.85 \pm 1.13$ & $3.87 \pm 1.02$ & $3.73 \pm 1.17$ & $4.15 \pm 1.24$ & 0.27 \\
\hline Serum triglycerides, $\mathrm{mmol} / \mathrm{L}$ & $1.52 \pm 0.67$ & $1.47 \pm 0.78$ & $1.56 \pm 0.85$ & $1.92 \pm 1.33^{*}$ & 0.04 \\
\hline Serum creatinine, $\mu \mathrm{mol} / \mathrm{L}$ & $69.2 \pm 14.8$ & $73.1 \pm 17.5$ & $75.0 \pm 16.2$ & $77.5 \pm 16.2^{*}$ & 0.04 \\
\hline Serum uric acid, $\mu \mathrm{mol} / \mathrm{L}$ & $313 \pm 70$ & $308 \pm 78$ & $320 \pm 72$ & $337 \pm 77$ & 0.17 \\
\hline Skin AGEs, arbitrary units & $1.61 \pm 0.16$ & $1.52 \pm 0.20$ & $2.20 \pm 0.27^{*, \dagger}$ & $2.22 \pm 0.30 *$ & $<0.0001$ \\
\hline Plasma AGE concentration, $\mu \mathrm{g} / \mathrm{mL}$ & $2.72[1.53-3.52]$ & $9.26[7.03-12.36]^{*}$ & $1.97[1.14-3.52]^{\dagger}$ & $7.98[6.56-11.97]^{*}$ & $<0.0001$ \\
\hline $\mathrm{cfPWV}, \mathrm{m} / \mathrm{s}$ & $7.71 \pm 1.22$ & $8.08 \pm 1.32$ & $7.87 \pm 1.23$ & $8.24 \pm 1.28^{*}$ & 0.05 \\
\hline
\end{tabular}

Values are presented as mean \pm standard deviation, median [interquartile range] or number of subjects (\%). For definitions of hypertension, diabetes, and prediabetes, see Methods. ${ }^{*} p<0.05$ vs. the group with both skin autofluorescence and plasma AGEs concentration below median. ${ }^{\dagger} p<0.05$ between the group with skin autofluorescence below median and plasma AGEs concentration above median and the group with skin autofluorescence above median and plasma AGE concentration below median. AF, autofluorescence; AGEs, advanced glycation end products; HDL, high-density lipoprotein; cfPWV, carotid-femoral pulse wave velocity.

cantly in age, proportions of men, current smoking and alcohol intake, prevalence of diabetes and prediabetes, and serum triglycerides and creatinine $(p \leq 0.04)$, with consistently higher levels in the group with both of the AGE measurements above the median. When the 2 groups with only 1 of the two AGE measurements above the median were compared, statistical difference was observed in current smoking and pulse rate $(p \leq 0.04)$.

We then performed continuous analyses to investigate correlates of these two AGE measurements. In stepwise multiple regression analyses, we considered sex, age, body mass index, current smoking and alcohol intake, fasting plasma glucose, serum total-to-HDL cholesterol ratio, serum triglycerides, serum creatinine and uric acid, the presence of diabetes or prediabetes, and the presence of hypertension. Skin autofluorescence was higher in men $(+17 \%, p=0.003)$ and those with advancing age $(+11 \%$ per 10 -year increase, $p<0.001)$, current smoking $(+18 \%, p=0.02)$ and diabetes or prediabetes $(+13 \%, p=0.09)$. Plasma AGE concentration was higher in men $(+33 \%, p<0.001)$ and those with diabetes or prediabetes $(+17 \%, p=0.03)$, advancing age $(+4 \%$ per 10 -year increase, $p=0.06)$, and currently smoking $(+14 \%, p=0.09$; Table 2).

\section{Relationship of cfPWV with Skin Autofluorescence and Plasma AGE Concentration}

Before adjustment, plasma AGE concentration was significantly associated with cfPWV $(r=0.15, p=0.02)$. This association was stronger $(p<0.001$ for interaction) in the presence $(r=0.42, p=0.02)$ than the absence $(r=0.08, p=0.28)$ of diabetes or prediabetes (Fig. 4$)$, and 
Liu et al.: A Comparative Study on Skin and Plasma Advanced Glycation End Products and Their Associations with Arterial Stiffness

Table 2. Multiple stepwise regression analyses on correlates of skin autofluorescence and plasma AGE concentration

Regression

coefficient $\pm \mathrm{SE}$

Skin autofluorescence (arbitrary units)

Age, +10 years

Male sex

Current smoking

Presence of diabetes or prediabetes

Plasma AGE concentration $(\mu \mathrm{g} / \mathrm{ml})$

Male sex

Presence of diabetes or prediabetes

Age, +10 years

Current smoking

$\begin{array}{lc}0.11 \pm 0.02 & <0.001 \\ 0.17 \pm 0.06 & 0.003 \\ 0.18 \pm 0.08 & 0.02 \\ 0.13 \pm 0.08 & 0.09 \\ & \\ 0.33 \pm 0.06 & <0.001 \\ 0.17 \pm 0.08 & 0.03 \\ 0.04 \pm 0.02 & 0.06 \\ 0.14 \pm 0.08 & 0.09\end{array}$

In a stepwise multiple linear regression model, we considered age, sex, body mass index, current smoking and alcohol intake, fasting plasma glucose, serum total-to-HDL, serum triglycerides, serum creatinine and uric acid, and the presence of hypertension and diabetes or prediabetes for entry and stay at a significance level of $p \leq 0.10$. For definitions of hypertension, diabetes, and prediabetes, see Methods. The variables are listed in the descending order of statistical significance. HDL, high-density lipoprotein; AGE, advanced glycation end product.

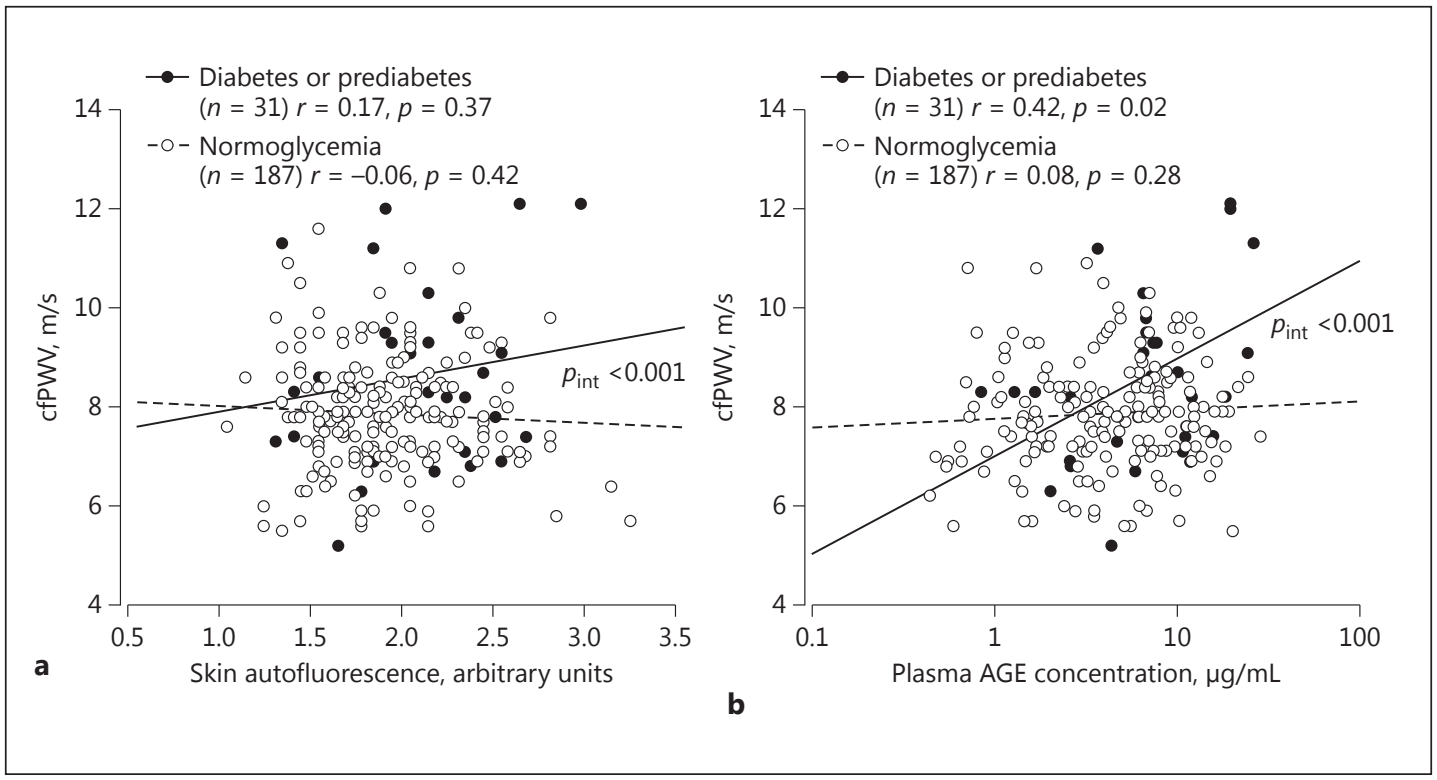

Fig. 4. Association of skin autofluorescence (a) and plasma advanced glycation end product (AGE) concentration (b), respectively, with carotid-femoral pulse wave velocity (cfPWV) according to the presence or absence of diabetes or prediabetes. A regression line was drawn. Correlation coefficients and their $p$ values are given for diabetes or prediabetes (dots with full line) and normoglycemia (circles with dashed line), separately. The $p$ value for the interaction between skin autofluorescence or plasma AGE concentration and the presence or absence of diabetes or prediabetes in relation to cfPWV is also given $\left(p_{\text {int }}\right)$. 
in sex-specific analyses, was significant in men $(r=0.22, p=0.03)$ but not women $(r=0.15$, $p=0.10$ ). After adjustment for sex, age, body mass index, current smoking and alcohol intake, mean arterial pressure, pulse rate, fasting plasma glucose, serum total-to-HDL cholesterol ratio, serum triglycerides, serum creatinine and uric acid, and the presence of hypertension, as appropriate, the results were confirmatory. For each 10 -fold increase in plasma AGE concentration, cfPWV increased in all subjects by $0.44 \mathrm{~m} / \mathrm{s}(p=0.04)$ and in subgroups of men and patients with diabetes and prediabetes by 0.51 and $3.01 \mathrm{~m} / \mathrm{s}(p \leq 0.02)$, respectively.

However, for skin autofluorescence, none of the associations with cfPWV reached statistical significance, either before or after adjustment, in all subjects or in various subgroups (Fig. $4 ; p \geq 0.37$ ).

\section{Discussion}

The main finding of our study was that skin autofluorescence and plasma AGE concentration were correlated with each other only in subjects with diabetes or prediabetes but not in those with normoglycemia. Nonetheless, the 2 AGE measurements had very similar determinants. In adjusted analyses, both measurements were higher with advancing age, in men than women, in patients with diabetes or prediabetes than subjects with normoglycemia, and in current smokers than nonsmokers. Plasma AGE concentration but not skin autofluorescence was positively associated with arterial stiffness in men and in subjects with diabetes or prediabetes.

AGE accumulation, as assessed by plasma AGE concentration, is a proven marker of arterial disease [8-12] and predictor of cardiovascular morbidity and mortality [5-7]. There is also some evidence that intervention of AGE formation may attenuate arterial lesions or reduce arterial stiffness [24, 25]. Skin autofluorescence as a measure of AGE accumulation is apparently a step forward in the assessment of AGEs in tissues. The condition is that this noninvasive device measurement of AGEs has to offer the same value of disease prediction as plasma AGE concentration.

An earlier study validated this skin autofluorescence method against skin biopsies on the same site of the arm for collagen-linked fluorescence and specific AGEs, such as pentosidine, carboxyethyl lysine, and CML [14]. In this particular study, skin autofluorescence was closely correlated with collagen-linked fluorescence and with the specific AGEs, with correlation coefficients greater than 0.40 . However, 2 subsequent studies did not show significant association between skin autofluorescence and plasma concentration of AGEs in patients with type 1 diabetic mellitus $(n=68)$ [20] or systemic lupus erythematosus $(n=9)$ [26]. These latter observations are in line with the overall results of our study. However, when plasma AGE concentration and skin autofluorescence were high, for instance, in the presence of type 2 diabetic mellitus in our study, skin autofluorescence was indeed significantly associated with plasma AGE concentration. In addition, a previous study found that skin autofluorescence was associated with the metabolic profile 10 years prior to, but not at the time of, the measurement [27], suggesting that skin autofluorescence is an indicator of long-term AGE accumulation in the body.

Our finding on the similarly elevated levels of skin autofluorescence and plasma AGE concentration in the presence of diabetes and prediabetes is in line with the results of several previous studies $[2,27,28]$, and may have straightforward explanations. With regard to gender difference, our current study and some previous studies showed higher levels of skin autofluorescence in men. However, other studies did not show gender difference [29] or even showed higher levels of AGEs in women [30,31]. In addition to inadequate power because of small sample size, several other factors might play a role. First, estrogen may be a protective 
Liu et al.: A Comparative Study on Skin and Plasma Advanced Glycation End Products and Their Associations with Arterial Stiffness

factor against AGE accumulation [32]. Although most (75\%) of our female participants were older than 45 years, estrogen protection in the early years of life may still show a difference in the levels of AGEs and even in the association with arterial stiffness at older age. Second, cigarette smoking can be a confounding factor. Cigarette smokers had higher levels of AGEs than nonsmokers in the present and previous studies [3,33]. Most of our male subjects were smokers. However, the gender difference in the levels of AGEs in our study remained statistically significant even after adjustment for current smoking. Third, skin color may influence the measurement of skin autofluorescence differently between men and women in the Chinese population. Dark skin color may cause autofluorescence measurement failure. Chinese men usually have darker color than Chinese women. In a Chinese methodological study, the frequency of measurement failures was higher in men than women $(21.5 \mathrm{vs.} 11.0 \%$, $p<0.01$ ) [29].

Why only plasma AGE concentration but not skin autofluorescence was associated with cfPWV is not understood. A speculative explanation is that plasma AGE concentration measures the AGEs in the circulation, and might therefore have a direct effect on blood vessels. However, a previous study that simultaneously investigated the relationship of skin autofluorescence and plasma AGE (CML) concentration with cfPWV in patients with type 1 diabetes mellitus showed a significant association for the former but not the latter measurement of AGEs [20]. In addition, even the studies with plasma AGE concentration produced contradictory results [8-12, 34]. In our previous population study, plasma AGE concentration was associated with arterial wave reflection [9] and pressure amplification in all subjects [12], but with cfPWV only in subjects older than 70 years [9]. In a study of 102 normoglycemic subjects, plasma CML concentration was not associated with cfPWV [34]. In our current study, a significant association for plasma AGE concentration was observed in patients with diabetes or prediabetes but not in subjects with normoglycemia. Most of the studies with skin autofluorescence showed a significant association with arterial stiffness [35-37]. However, the possibility of publication bias with this relatively new technique cannot be entirely excluded.

Our study has to be interpreted within the context of its limitations. First, our study is cross-sectional and therefore does not allow us to draw any causal inference. Second, our study had a relatively small sample size and might be inadequately powered to detect weak association between skin autofluorescence and cfPWV.

In conclusion, although skin and plasma AGEs were similarly elevated in men and in the presence of diabetes or prediabetes, they might measure something different and have different clinical relevance, such as for arterial stiffness.

\section{Acknowledgments}

We gratefully acknowledge the voluntary participation of all study subjects and the technical assistance of Jun-Wei Li, Xiao-Ren Lin, Bei-Wen Lv, Yu-Zhong Shi, Jie Wang, Yi Zhou, and Yi-Ni Zhou (The Shanghai Institute of Hypertension, China). The present study was financially supported by grants from: the National Natural Science Foundation of China (grants 81170245, 81270373, 81470533, and 81400312), the Ministry of Science and Technology (grant 2013CB530700 and a grant for China-European Union collaborations [1012]), and the Ministry of Education (NCET-09-0544), Beijing, China; the Shanghai Commissions of Science and Technology (grants 11QH1402000, 14ZR1436200, and 15XD1503200) and Education (grant JDY09088), the Shanghai Bureau of Health (grants XBR2011004, 20101051, and 20144Y0213), and Shanghai Jiaotong University School of Medicine (grant 14XJ10071).

\section{Disclosure Statement}

The authors declared no conflict of interest. 
Liu et al.: A Comparative Study on Skin and Plasma Advanced Glycation End Products and Their Associations with Arterial Stiffness

\section{References}

1 Basta G, Schmidt AM, De Caterina R: Advanced glycation end products and vascular inflammation: implications for accelerated atherosclerosis in diabetes. Cardiovasc Res 2004;63:582-592.

2 Goh SY, Cooper ME: Clinical review: the role of advanced glycation end products in progression and complications of diabetes. J Clin Endocrinol Metab 2008;93:1143-1152.

3 Cerami C, Founds H, Nicholl I, Mitsuhashi T, Giordano D, Vanpatten S, Lee A, Al-Abed Y, Vlassara H, Bucala R, Cerami A: Tobacco smoke is a source of toxic reactive glycation products. Proc Natl Acad Sci USA 1997; 94 : 13915-13920.

4 Luevano-Contreras C, Chapman-Novakofski K: Dietary advanced glycation end products and aging. Nutrients 2010;2:1247-1265.

5 Nin JW, Jorsal A, Ferreira I, Schalkwijk CG, Prins MH, Parving HH, Tarnow L, Rossing P, Stehouwer CD: Higher plasma levels of advanced glycation end products are associated with incident cardiovascular disease and allcause mortality in type 1 diabetes: a 12-year follow-up study. Diabetes Care 2011;34:442-447.

6 Kilhovd BK, Juutilainen A, Lehto S, Ronnemaa T, Torjesen PA, Birkeland KI, Berg TJ, Hanssen KF, Laakso M: High serum levels of advanced glycation end products predict increased coronary heart disease mortality in nondiabetic women but not in nondiabetic men: a population-based 18-year follow-up study. Arterioscler Thromb Vasc Biol 2005;25:815-820.

7 Kilhovd BK, Juutilainen A, Lehto S, Ronnemaa T, Torjesen PA, Hanssen KF, Laakso M: Increased serum levels of advanced glycation endproducts predict total, cardiovascular and coronary mortality in women with type 2 diabetes: a population-based 18 year follow-up study. Diabetologia 2007;50:1409-1417.

8 McNulty M, Mahmud A, Feely J: Advanced glycation end-products and arterial stiffness in hypertension. Am J Hypertens 2007;20:242-247.

9 Huang QF, Sheng CS, Liu M, Li FH, Li Y, Wang JG: Arterial stiffness and wave reflections in relation to plasma advanced glycation end products in a Chinese population. Am J Hypertens 2013;26:754-761.

10 Semba RD, Najjar SS, Sun K, Lakatta EG, Ferrucci L: Serum carboxymethyl-lysine, an advanced glycation end product, is associated with increased aortic pulse wave velocity in adults. Am J Hypertens 2009;22:74-79.

11 Won KB, Chang HJ, Park SH, Hong SY, Jang Y, Chung N: High serum advanced glycation end-products predict coronary artery disease irrespective of arterial stiffness in diabetic patients. Kor Circ J 2012;42:335-340.

12 Huang QF, Sheng CS, Kang YY, Zhang L, Wang S, Li FK, Cheng YB, Guo QH, Li Y, Wang JG: Central and peripheral blood pressures in relation to plasma advanced glycation end products in a Chinese population. J Hum Hypertens 2016;30:430-435.

13 Bohlender JM, Franke S, Stein G, Wolf G: Advanced glycation end products and the kidney. Am J Physiol Renal Physiol 2005;289:F645-F659.

14 Meerwaldt R, Graaff R, Oomen PH, Links TP, Jager JJ, Alderson NL, Thorpe SR, Baynes JW, Gans RO, Smit AJ: Simple non-invasive assessment of advanced glycation endproduct accumulation. Diabetologia 2004;47: 1324-1330.

15 de Vos LC, Noordzij MJ, Mulder DJ, Smit AJ, Lutgers HL, Dullaart RP, Kamphuisen PW, Zeebregts CJ, Lefrandt JD: Skin autofluorescence as a measure of advanced glycation end products deposition is elevated in peripheral artery disease. Arterioscler Thromb Vasc Biol 2013;33:131-138.

16 Wang AY, Wong CK, Yau YY, Wong S, Chan IH, Lam CW: Skin autofluorescence associates with vascular calcification in chronic kidney disease. Arterioscler Thromb Vasc Biol 2014;34:1784-1790.

17 Araszkiewicz A, Naskret D, Zozulinska-Ziolkiewicz D, Pilacinski S, Uruska A, Grzelka A, Wegner M, WieruszWysocka B: Skin autofluorescence is associated with carotid intima-media thickness, diabetic microangiopathy, and long-lasting metabolic control in type 1 diabetic patients. Results from Poznan Prospective Study. Microvasc Res 2015;98:62-67.

18 de Vos LC, Mulder DJ, Smit AJ, Dullaart RP, Kleefstra N, Lijfering WM, Kamphuisen PW, Zeebregts CJ, Lefrandt JD: Skin autofluorescence is associated with 5-year mortality and cardiovascular events in patients with peripheral artery disease. Arterioscler Thromb Vasc Biol 2014;34:933-938.

19 Siriopol D, Hogas S, Veisa G, Mititiuc I, Volovat C, Apetrii M, Onofriescu M, Busila I, Oleniuc M, Covic A: Tissue advanced glycation end products (AGEs), measured by skin autofluorescence, predict mortality in peritoneal dialysis. Int Urol Nephrol 2015;47:563-569.

20 Llaurado G, Ceperuelo-Mallafre V, Vilardell C, Simo R, Gil P, Cano A, Vendrell J, Gonzalez-Clemente JM: Advanced glycation end products are associated with arterial stiffness in type 1 diabetes. J Endocrinol 2014;221:405413.

21 Wei FF, Li Y, Zhang L, Xu TY, Ding FH, Staessen JA, Wang JG: Association of target organ damage with 24-hour systolic and diastolic blood pressure levels and hypertension subtypes in untreated Chinese. Hypertension 2014;63:222-228.

22 Wei FF, Li Y, Zhang L, Xu TY, Ding FH, Wang JG, Staessen JA: Beat-to-beat, reading-to-reading, and day-to-day blood pressure variability in relation to organ damage in untreated Chinese. Hypertension 2014;63:790-796.

23 O’Brien E, Asmar R, Beilin L, Imai Y, Mancia G, Mengden T, Myers M, Padfield P, Palatini P, Parati G, Pickering T, Redon J, Staessen J, Stergiou G, Verdecchia P: Practice guidelines of the European Society of Hypertension for clinic, ambulatory and self blood pressure measurement. J Hypertens 2005;23:697-701.

24 Kass DA, Shapiro EP, Kawaguchi M, Capriotti AR, Scuteri A, deGroof RC, Lakatta EG: Improved arterial compliance by a novel advanced glycation end-product crosslink breaker. Circulation 2001;104:1464-1470. 
Liu et al.: A Comparative Study on Skin and Plasma Advanced Glycation End Products and Their Associations with Arterial Stiffness

25 Satheesan S, Figarola JL, Dabbs T, Rahbar S, Ermel R: Effects of a new advanced glycation inhibitor, LR-90, on mitigating arterial stiffening and improving arterial elasticity and compliance in a diabetic rat model: aortic impedance analysis. Br J Pharmacol 2014;171:3103-3114.

26 Nienhuis HL, de Leeuw K, Bijzet J, Smit A, Schalkwijk CG, Graaff R, Kallenberg CG, Bijl M: Skin autofluorescence is increased in systemic lupus erythematosus but is not reflected by elevated plasma levels of advanced glycation endproducts. Rheumatology (Oxford) 2008;47:1554-1558.

27 Rajaobelina K, Cougnard-Gregoire A, Delcourt C, Gin H, Barberger-Gateau P, Rigalleau V: Autofluorescence of skin advanced glycation end products: marker of metabolic memory in elderly population. J Gerontol A Biol Sci Med Sci 2015;70:841-846.

28 Lutgers HL, Graaff R, Links TP, Ubink-Veltmaat LJ, Bilo HJ, Gans RO, Smit AJ: Skin autofluorescence as a noninvasive marker of vascular damage in patients with type 2 diabetes. Diabetes Care 2006;29:2654-2659.

29 Yue X, Hu H, Koetsier M, Graaff R, Han C: Reference values for the Chinese population of skin autofluorescence as a marker of advanced glycation end products accumulated in tissue. Diabet Med 2011;28:818-823.

30 Sebekova K, Krivosikova Z, Gajdos M: Total plasma Nepsilon-(carboxymethyl)lysine and sRAGE levels are inversely associated with a number of metabolic syndrome risk factors in non-diabetic young-to-middle-aged medication-free subjects. Clin Chem Lab Med 2014;52:139-149.

31 Ahmad MS, Damanhouri ZA, Kimhofer T, Mosli HH, Holmes E: A new gender-specific model for skin autofluorescence risk stratification. Sci Rep 2015;5:10198.

32 Pereira-Simon S, Rubio GA, Xia X, Cai W, Choi R, Striker GE, Elliot SJ: Inhibition of advanced glycation end products (AGEs) accumulation by pyridoxamine modulates glomerular and mesangial cell estrogen receptor $\alpha$ expression in aged female mice. PLoS One 2016;11:e0159666.

33 Koetsier M, Lutgers HL, de Jonge C, Links TP, Smit AJ, Graaff R: Reference values of skin autofluorescence. Diabetes Technol Ther 2010;12:399-403.

34 Baumann M, Richart T, Sollinger D, Pelisek J, Roos M, Kouznetsova T, Eckstein HH, Heemann U, Staessen JA: Association between carotid diameter and the advanced glycation end product N-epsilon-carboxymethyllysine (CML). Cardiovasc Diabetol 2009;8:45.

35 Ueno H, Koyama H, Tanaka S, Fukumoto S, Shinohara K, Shoji T, Emoto M, Tahara H, Kakiya R, Tabata T, Miyata T, Nishizawa Y: Skin autofluorescence, a marker for advanced glycation end product accumulation, is associated with arterial stiffness in patients with end-stage renal disease. Metabolism 2008;57:1452-1457.

36 Watfa G, Soulis G, Tartagni E, Kearney-Schwartz A, Borghi C, Salvi P, Benetos A: Relationship between tissue glycation measured by autofluorescence and pulse wave velocity in young and elderly non-diabetic populations. Diabetes Metab 2012;38:413-419.

37 Hofmann B, Adam AC, Jacobs K, Riemer M, Erbs C, Bushnaq H, Simm A, Silber RE, Santos AN: Advanced glycation end product associated skin autofluorescence: a mirror of vascular function? Exp Gerontol 2013;48: 38-44. 